\title{
UNIT GROUPS OF COMPLETED MODULAR GROUP ALGEBRAS AND THE ISOMORPHISM PROBLEM
}

\author{
FRANK RÕHL
}

(Communicated by Warren J. Wong)

\begin{abstract}
In this paper it is shown that isomorphism of group algebras of finite $p$-groups over the field of $p$ elements implies isomorphism of the groups, if one of the groups has a normal complement in the group of normalized units of the group algebra. Furthermore, a class of groups satisfying this condition is provided, and it is shown that the associated graded Lie-p-algebra of the group of normalized units of the Magnus algebra of $G, G$ being a residually 'nilpotent $p$-group of bounded exponent', is a split extension of the one associated to $G$.
\end{abstract}

\section{INTRODUCTION}

Before the isomorphism problem for integral group rings of finite nilpotent groups was solved, there was much interest in finding groups that have a normal complement in the group of units of its integral group ring. Part of this interest was certainly due to the fact that these groups were characterized by their integral group ring.

In the modular case, it is still an open problem whether a finite $p$-group $G$ admitting a normal complement in the group of units of its group algebra $\mathbf{F}_{p} G, \mathbf{F}_{p}$ denoting the field of $p$ elements, is characterized by $\mathbf{F}_{p} G$. Despite all efforts, this is known to be true only for a rather small class of groups (see [11, p. 274] for a brief summary, which is still up to date). In the second part of this paper we show that the above problem has a positive solution in general. The proof makes use of graded techniques and is centered around the group algebra of the group $U_{1}$ of normalized units of $\mathbf{F}_{p} G$ rather than around $\mathbf{F}_{p} G$ itself.

Together with the groups known to have a normal complement, this result gives a rather impressive class of concrete groups, which are characterized by their modular group algebra: Every finite $p$-Sylow subgroup of a unit group of an $\mathbf{F}_{p}$-algebra, every circle group of a finite-dimensional nilpotent $\mathbf{F}_{p}$-algebra, and every normal subgroup of the group of unitriangular $n \times n$ matrices over $\mathbf{F}_{p}$ belongs to this class.

Received by the editors July 10, 1989 and, in revised form, April 6, 1990.

1980 Mathematics Subject Classification (1985 Revision). Primary 16A25, 16A26; Secondary $16 \mathrm{~A} 03$. 
Although the statement that each finite $p$-group is normally complemented in $U_{1}$ is definitely wrong (the first counterexample-the dihedral group of order 16-was obtained by the use of a computer [5]; another was obtained in [1]), we provide in the first part of this paper some evidence that it might not be far from the truth. At least the graded Lie $p$-algebra associated to $G$ admits the analogue of a normal complement (namely a complementary Lie $p$-ideal) in the one associated to $U_{1}$ (the filtration is taken with respect to modular dimension subgroups both for $G$ and $U_{1}$ ). And this holds even for $G$ being residually a nilpotent $p$-group of bounded exponent and $U_{1}$ the group of normalized units of the Magnus algebra of $G$ over $\mathbf{F}_{p}$.

More or less in the course of proving this latter result, we find that the intersection of $G$ with the modular dimension subgroups of $U_{1}$ yields just the modular dimension subgroups of $G$, which generalizes a result of Moran [4] and answers a question raised by Sandling in a private communication.

It should be mentioned that many of these issues originate in [13].

\section{A SPLITTING THEOREM}

We start our investigations by generalizing some of Johnson's observations concerning finite $p$-groups in [2] to the case where $G$ is residually a nilpotent $p$-group of bounded exponent, which will be assumed to be the case throughout the entire section.

Denote by $\overline{\mathbf{F}_{p} G}:=\operatorname{Lim}_{\leftarrow--} G / \Delta G^{n}$ the Magnus algebra of $G$ over $\mathbf{F}_{p}$, where $\Delta G$ is the augmentation ideal of $\mathbf{F}_{p} G$. Let $U_{1}$ be the group of normalized units of $\overline{\mathbf{F}_{p} G}$. The Jacobson radical of $\overline{\mathbf{F}_{p} G}$ coincides with the augmentation ideal $\bar{\Delta} \bar{G}$ of $\overline{\mathrm{F}_{p} G}$. Hence, $U_{1}$ is the multiplicative group $1+\bar{\Delta} \bar{G}$. Note that $G$ embeds into $\overline{\mathrm{F}_{p} G}$ and thus into $U_{1}$, since $\cap_{n} \Delta G^{n}=0$ (see, for instance [6]). Further on, since the kernel of the projection $\overline{\mathbf{F}_{p} G} \rightarrow \mathbf{F}_{p} G / \Delta G^{n}$ turns out to be $\bar{\Delta} \bar{G}^{n}$, we are allowed to identify $\operatorname{gr}_{p} \overline{\mathbf{F}_{p}}:=\bigoplus_{i \geq 0} \bar{\Delta} \bar{G}^{i} / \bar{\Delta} \bar{G}^{i+1}$ with gr $\mathrm{F}_{p} G$, and for the $n$th modular dimension subgroup $M_{n}(G)$ of $G$ we obtain $M_{n}(G):=G \cap\left(1+\Delta G^{n}\right)=G \cap\left(1+\bar{\Delta} \bar{G}^{n}\right)$.

The natural embedding $G \subset U_{1}$ extends to an $\mathbf{F}_{p}$-algebra homomorphism $\bar{l}: \overline{\mathbf{F}_{p} G} \rightarrow \overline{\mathbf{F}_{p} U_{1}}$, whereas the universal property of $\overline{\mathbf{F}_{p} U_{1}}$ together with $U_{1} \subset$ $\overline{\mathbf{F}_{p} G}$ provides an $\mathbf{F}_{p}$-algebra homomorphism $\bar{\pi}: \overline{\mathbf{F}_{p} U_{1}} \rightarrow \overline{\mathbf{F}_{p} G}$ (note that $\mathbf{F}_{p} G \rightarrow \overline{\mathbf{F}_{p} U_{1}}$ resp. $\mathbf{F}_{p} U_{1} \rightarrow \overline{\mathbf{F}_{p} G}$ are continuous, since we are concerned with algebra homomorphisms and the $\Delta$-adic topology). This far we have the following situation:

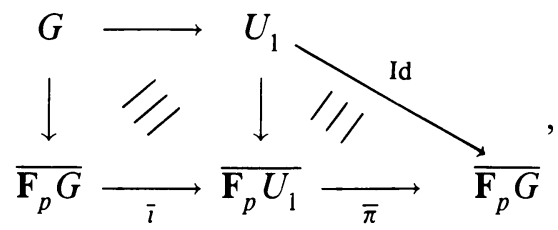

and by the definition of the two bottom maps, their composite is just the identity 
on $\overline{\mathbf{F}_{p} G}$. Hence

$$
\overline{\mathbf{F}_{p} U_{1}} \tilde{\sim} \overline{\mathbf{F}_{p} G} \oplus \operatorname{ker} \bar{\pi}
$$

(see also [2]).

Regrettably, this does not in general imply that $G$ already admits a normal complement in $U_{1}$ (see $[1,5]$ for counterexamples and recall that for finite $p$-groups $G$, one has $\left.\overline{\mathbf{F}_{p} G}=\mathbf{F}_{p} G\right)$. But in the graded situation this turns out to be true.

Before we come to this, we need one more observation that is of interest in its own right, since it answers a question raised by Sandling in a private communication and generalizes the well-known fact that the Frattini subgroup of $G$ coincides with the intersection of $G$ with the Frattini subgroup of $U_{1}$ (see [4] and also [13] for related questions).

Let $M_{n}\left(U_{1}\right)=\left(1+\Delta U_{1}^{n}\right) \cap U_{1}$ be the $n$th modular dimension subgroup of $U_{1}$. Then

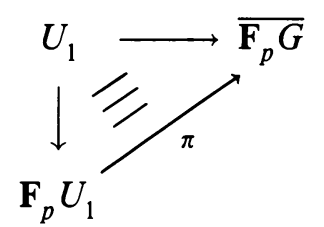

together with the fact that $\pi\left(\Delta U_{1}^{n}\right) \subset \bar{\Delta} \bar{G}^{n}$ shows $M_{n}\left(U_{1}\right) \subset 1+\bar{\Delta} \bar{G}^{n}$ for all $n$. Thus $M_{n}\left(U_{1}\right) \cap G \subset\left(1+\bar{\Delta} \bar{G}^{n}\right) \cap G=M_{n}(G)$. The other inclusion being trivial (for example by means of an embedding $\mathbf{F}_{p} G \subset \overline{\mathbf{F}_{p} G} \hookrightarrow \overline{\mathbf{F}_{p} U_{1}}$ ), we obtain the following:

2.2. Proposition. $M_{n}\left(U_{1}\right) \cap G=M_{n}(G)$ for all $n$.

(2.2) implies in particular that we may identify $\operatorname{gr} G:=\bigoplus_{i \geq 1} M_{i}(G) / M_{i+1}(G)$ with its image in $\operatorname{gr} U_{1}$ (induced by $G \subset U_{1}$ ) via the natural isomorphism.

$$
M_{i}(G) \cdot M_{i+1}\left(U_{1}\right) / M_{i+1}\left(U_{1}\right) \stackrel{\sim}{\rightarrow} M_{i}(G) / G \cap M_{i+1}\left(U_{1}\right) .
$$

Because $M_{i}\left(U_{1}\right) \subset 1+\bar{\Delta} \bar{G}^{i}$, the inclusion $U_{1} \subset \overline{\mathbf{F}_{p} G}$ induces a homomorphism of Lie $p$-algebras

given by

$$
\hat{\pi}: \operatorname{gr} U_{1} \rightarrow \operatorname{gr} \mathbf{F}_{p} G
$$

$$
u M_{n+1}\left(U_{1}\right) \mapsto u-1+\Delta G^{n+1} .
$$

(Recall that we identified $\operatorname{gr}_{\bar{p}} G$ with $\operatorname{gr}_{p} G$.) Note that although this map is induced by $U_{1}=1+\bar{\Delta} \bar{G} \subset \overline{\mathbf{F}_{p} G}$, it is far from being an inclusion because the filtration $\left(\bar{\Delta} \bar{G}^{n}\right)_{n \in \mathbb{N}}$ of $\overline{\mathbf{F}_{p} G}$ does not coincide with the filtration $\left(M_{n}\left(U_{1}\right)\right)_{n \in \mathbb{N}}$ of $U_{1}$.

Now we can state the following:

\subsection{Theorem.}

$$
\operatorname{gr} U_{1} \stackrel{\sim}{\rightarrow} \operatorname{gr} G \oplus \operatorname{ker} \hat{\pi}
$$


Proof. (2.1) induces

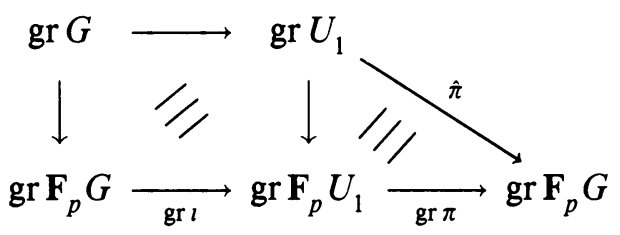

where the left vertical map is given by $g M_{n+1}(G) \mapsto g-1+\Delta G^{n+1}$, and analogously for $\operatorname{gr} U_{1}$. Let us denote the embedding $\operatorname{gr} G \rightarrow \operatorname{gr} U_{1}$ given by $g M_{n+1}(G) \mapsto g M_{n+1}\left(U_{1}\right)$ by $\hat{\imath}$.

Now, $\bar{\pi}$ being a surjective $\mathbf{F}_{p}$-algebra, homomorphism gives gr $\pi\left(\Delta U_{1} / \Delta U_{1}^{2}\right)$ $=\Delta G / \Delta G^{2} \tilde{\rightarrow} G / M_{2}(G)$, the last isomorphism being given by $g-1+\Delta G^{2} \mapsto$ $g M_{2}(G)$; and in the same way one has $U_{1} / M_{2}\left(U_{1}\right) \sim \Delta U_{1} / \Delta U_{1}^{2}$. But the deg 1 elements of $\operatorname{gr} U_{1}$ generate $\operatorname{gr} U_{1}$ as a Lie $p$-algebra [7]. Thus $\hat{\pi}$ maps generators of $\operatorname{gr} U_{1}$ into the image of $\operatorname{gr} G$ in $\operatorname{gr}_{p} G$, whence $\hat{\pi}$ factors through gr $G$ :

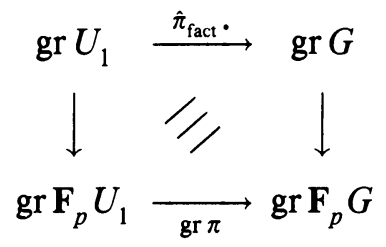

Since $\mathrm{gr}$ is a functor and $\overline{\pi l}=\mathrm{Id}$, one has $\operatorname{gr} \pi \operatorname{gr} l=\mathrm{Id}$. By the injectivity of the vertical maps of the last diagram, this implies

$$
\hat{\pi}_{\text {fact }} \cdot \hat{\imath}=\mathrm{Id}
$$

and $\operatorname{ker} \hat{\pi}=\operatorname{ker} \hat{\pi}_{\text {fact }}$. gives the assertion. Q.E.D.

Remark. Of course one would like to have such a theorem for the integral situation, too. The difficulties are as follows: Either one replaces $\operatorname{gr} G$ by the Lie ring associated to the lower central series of $G$, in which case one would sacrifice the injectivity of $\operatorname{gr} G \hookrightarrow \operatorname{gr} \overline{F_{p} G}$, since not all groups have the dimension subgroup property; or one replaces gr $G$ by the Lie ring associated to the integral dimension subgroup series of $G$, in which case it is not known whether this Lie ring is still generated by deg 1 elements [7].

In view of the result of the next section, we are now going to supply some normally complemented groups.

We start with a possibly well-known observation: If $U$ denotes the group of all units of $\mathrm{F}_{p} G, G$ a finite $p$-group, and $U_{1}$ that of normalized units of $\mathbf{F}_{p} G$, then $G$ has a normal complement in $U$ if and only if it has one in $U_{1}$. (The "if" part follows immediately from $U=\mathbf{F}_{p}^{*} \times U_{1}, \mathbf{F}_{p}^{*}$ being the group of units of $\mathbf{F}_{p}$. And, for the other direction, note that $U_{1}$ is a finite $p$-group and thus nilpotent. Hence $U=\mathbf{F}_{p}^{*} \times U_{1}$ and all normal complements $N$ of $G$ in $U$, too, are nilpotent. Since $N$ then is the direct product of its $q$-Sylow subgroups, 
$q$ a prime, and $p-1=\# \mathrm{~F}_{p}^{*}$ divides the order of $N$, the $p$-Sylow subgroup of $N$ provides a normal complement of $G$ in $U_{1}$.)

The following theorem is a slight generalization of results contained in [2] (see also [11] from the integral case).

2.4. Theorem. For $G$ a finite $p$-group and $U_{1}$ the group of normalized units of $\mathbf{F}_{p} G$, there exists an $N \triangleleft U_{1}$ such that $U_{1}=G \propto N$, if

(i) $G$ is the circle group of a finite-dimensional nilpotent $\mathbf{F}_{p}$-algebra (see [2, Theorem 5]), or

(ii) $G$ is a finite $p$-Sylow subgroup of a unit group of an $\mathbf{F}_{p}$-algebra (see [2, Theorem 6]).

Proof. (i) Let $G=(A, \circ), A$ a nilpotent $\mathbf{F}_{p}$-algebra of finite dimension, and $x \circ y:=x+y+x y$ for $x, y \in A$. Then one has

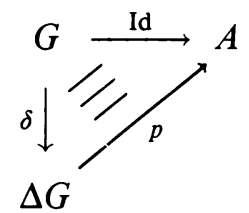

with an injective group homomorphism $\delta: G \rightarrow(\Delta G, \circ)$ given by $\delta(g)=$ $g-1 ; \operatorname{ker} p$ being an ideal of $\Delta G$, forms a normal subgroup of $(\Delta G, \circ)$ and $\delta G \cap \operatorname{ker} p=0$. Thus $(\Delta G, \circ)=\delta G \propto \operatorname{ker} p$, and $U_{1} \stackrel{\rightarrow}{\rightarrow}(\Delta G, \circ)$ by $u \mapsto u-1$.

(ii) Let $G$ be a finite $p$-Sylow subgroup of the unit group of the $\mathbf{F}_{p}$-algebra $A$. Then

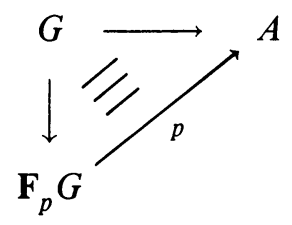

$U_{1}$ being a finite $p$-group containing $G$, is mapped under $p$ into a finite $p$-group containing $G$. By the maximality of $p$-Sylow subgroups, we obtain $p\left(U_{1}\right) \subset G$, and the above diagram shows that $G \rightarrow U_{1} \stackrel{p}{\longrightarrow} G$ is the identity. Thus $1+\operatorname{ker} p$ provides a normal complement of $G$ in $U_{1}$. Q.E.D.

One more result should be mentioned in this connection: Let $U_{n}\left(\mathbf{F}_{p}\right)$ be the group of unitriangular $n \times n$ matrices over $\mathbf{F}_{p}$ (1's on the diagonal, 0's above). Then $U_{n}\left(\mathbf{F}_{p}\right)$ is isomorphic to the circle group of the (finite-dimensional, nilpotent) $\mathbf{F}_{p}$-algebra of strictly lower triangular matrices. In [3], Levchuk has shown that every normal subgroup of this circle group is already a subring. Thus Theorem 2.4(i) implies the following:

2.5. Corollary. Every normal subgroup $N$ of $U_{n}\left(\mathbf{F}_{p}\right)$ has a normal complement in its group of normalized units of $\mathbf{F}_{p} N$. 


\section{THE ISOMORPHISM PROBLEM FOR COMPLEMENTED GROUPS}

Let $G$ be residually a nilpotent $p$-group of bounded exponent and $U_{1}$ be the group of normalized units of $\overline{\mathrm{F}_{p} G}$. Then we have the following lemma, which is of interest in its own right.

3.1. Lemma. $U_{1}$ is residually a nilpotent p-group of bounded exponent.

Proof. Since a completion of a Hausdorff space is Hausdorff again, and the topology of $\overline{\mathbf{F}_{p} G}$ coincides with that defined by taking $\left(\bar{\Delta} \bar{G}^{n}\right)_{n \in \mathbb{N}}$ as a fundamental system of neighborhoods of 0 , one has $\cap \bar{\Delta} \bar{G}^{n}=0$. Because of $M_{n}\left(U_{1}\right) \subset 1+\bar{\Delta} \bar{G}^{n}$, this implies $\cap M_{n}\left(U_{1}\right)=1 . M_{n}\left(U_{1}\right)=\prod \gamma_{i}\left(U_{1}\right)^{p^{j}}$, where the product is taken over all $\left(i, p^{j}\right)$ with $i \cdot p^{j} \geq n$ and $\gamma_{i}\left(U_{1}\right)$ being the $i$ th term of the lower central series of $U_{1}$, gives the result. Q.E.D.

We adapt Sandling's definition of a group base to our situation: A subgroup $H$ of $U_{1}$ forms a group base of $\overline{\mathbf{F}_{p} G}$ if the inclusion $H \subset U_{1}$ can be extended to an isomorphism $\overline{\mathbf{F}_{p} H} \dot{\rightarrow \mathbf{F}_{p} G}$. We thus may (and will) identify $\overline{\mathbf{F}_{p} G}$ with $\overline{\mathbf{F}_{p} H}$ for group bases $H$ of $\overline{\mathbf{F}_{p} G}$. We now have the following theorem:

3.2. Theorem. Let $G, H$, and $U_{1}$ be as above. If $U_{1}=G \propto N$ for some normal subgroup $N$ of $U_{1}$, then $H \cap N=1$.

Proof. Assuming $1 \neq h \in H \cap N$, there exists a positive integer $n$ with $h \in$ $M_{n}(H) \backslash M_{n+1}(H)$, by the preceding lemma.

The inclusion $G \subset U_{1}$ and the projection $p: U_{1} \rightarrow G$ with kernel $N$ and being the identity on $G$ induce (analogously to (2.1))

$$
\overline{\mathbf{F}_{p} G} \stackrel{i}{\longrightarrow} \overline{\mathbf{F}_{p} U_{1}} \stackrel{\bar{p}}{\rightarrow} \overline{\mathbf{F}_{p} G}
$$

respectively, for the graded situation,

$$
\mathrm{gr} \overline{\mathbf{F}_{p} G} \stackrel{\mathrm{gr} \bar{i}}{\longrightarrow} \mathrm{gr} \overline{\mathbf{F}_{p} U_{1}} \stackrel{\mathrm{gr} \bar{p}}{\longrightarrow} \mathrm{gr}_{\overline{\mathbf{F}_{p} G}},
$$

with gr $\bar{p}$ being given by $u-1+\bar{\Delta} \bar{U}_{1}^{n} \mapsto p(u)-1+\bar{\Delta} \bar{G}^{n}$. Since $\overline{p l}=\mathrm{Id}$, one has $\operatorname{gr} \bar{p} \operatorname{gr} \bar{l}=\mathrm{Id}$.

On the other hand, both $\operatorname{gr} G$ and $\operatorname{gr} H$ embed into $\operatorname{gr} \overline{\mathbf{F}_{p} G}$, and their images coincide, since $\mathrm{gr}_{1} \overline{\mathrm{F}_{p} G}=\bar{\Delta} \bar{G} / \bar{\Delta} \bar{G}^{2} \tilde{\rightarrow} G / M_{2}(G)$ by our identification of $\bar{\Delta} \bar{G} / \bar{\Delta} \bar{G}^{2}$ with $\Delta G / \Delta G^{2}$ (and analogously for $\operatorname{gr}_{1} \overline{\mathbf{F}_{p} H}$ ) and $G / M_{2}(G)$ (resp., $H / M_{2}(H)$ ) generates gr $G$ (resp., gr $H$ ) as a Lie $p$-algebra. Hence there exists $g \in G$ with

$$
g-1+\bar{\Delta} \bar{G}^{n+1}=h-1+\bar{\Delta} \bar{G}^{n+1} .
$$

Furthermore, since $h \in M_{n}(H) \backslash M_{n+1}(H)$, and thus $h-1 \in \bar{\Delta} \bar{G}^{n} \backslash \bar{\Delta} \bar{G}^{n+1}$, one has $g \in M_{n}(G) \backslash M_{n+1}(G)$. 
Now, on the one hand $\operatorname{gr} \bar{p} \operatorname{gr} \bar{l}\left(h-1+\bar{\Delta} \bar{G}^{n+1}\right)=\operatorname{gr} \bar{p} \operatorname{gr} \bar{l}\left(g-1+\bar{\Delta} \bar{G}^{n+1}\right)=$ $g-1+\bar{\Delta} \bar{G}^{n+1}$, and on the other hand

$$
\operatorname{gr} \bar{p}\left(h-1+\bar{\Delta} \bar{U}_{1}^{n+1}\right)=p(h)-1+\bar{\Delta} \bar{G}^{n+1}=\bar{\Delta}_{\bar{G}}^{n+1}
$$

by the choice of $h \in \operatorname{ker} p=N$.

Thus, $g \in M_{n+1}(G)$, a contradiction. Q.E.D.

Thus in the situation of the Theorem, the natural projection $U_{1} \rightarrow G$ gives a monomorphism $H \hookrightarrow G$. If we require $G$ to be a nilpotent $p$-group of bounded exponent, i.e., $M_{n}(G)=1$ for some $n$, then the above monomorphism turns out already to be an isomorphism, for it induces the same isomorphism $\mathrm{gr} H \stackrel{\sim}{\rightarrow} \mathrm{gr} G$ as the one coming from the coincidence of generators of the images of $\mathrm{gr} H$ and $\operatorname{gr} G$ in $\overline{\mathbf{F}_{p} G}$, and a simple induction gives the result. Thus the following:

3.3. Corollary. If $G$ is a nilpotent p-group of bounded exponent, normally complemented in the group of normalized units of $\overline{\mathbf{F}_{p} G}$, then $G$ is characterized by $\overline{\mathbf{F}_{p} G}$.

Recall that for finite $p$-groups $G$, one has $\mathbf{F}_{p} G=\overline{\mathbf{F}_{p} G}$, and $U_{1}$ is just the group of normalized units of $\mathbf{F}_{p} G$. Hence, if $U_{1}=G \propto N$, then $G$ is characterized by $\mathbf{F}_{p} G$. In particular, the groups treated in (2.4) and (2.5) are characterized by $\mathbf{F}_{p} G$. This generalizes the results in $[8$, Theorem 12, p. 318] and [9] considerably. As another application, one obtains from Sandling's results that finite $p$-groups $G$ with $\gamma_{2}(G)^{p} \cdot \gamma_{3}(G)=1$ are normally complemented in $U$, without further proof that these groups are characterized by $\mathbf{F}_{p} G$ (see $[12,1.1$ Theorem and 1.2 Theorem]).

\section{REFERENCES}

1. L. R. Ivory, A note on normal complements in $\bmod p$ envelopes, Proc. Amer. Math. Soc. 79 (1980), 9-12.

2. D. L. Johnson, The modular group ring of a finite p-group, Proc. Amer. Math. Soc. 68 (1978), 19-22.

3. V. M. Levchuk, Relation of the unitriangular group to certain rings, Algebra and Logic $\mathbf{1 5}$ (1976), 348-360. (translation)

4. L. E. Moran, The modular group ring of a p-group, M. Phil. Thesis, University of Nottingham, 1972.

5. L. E. Moran and R. N. Tench, Normal complements in mod p-envelopes, Israel J. Math. 27 (1977), 331-338.

6. I. B. S. Passi, Group rings and their augmentation ideals, Lecture Notes in Math., vol. 715, Springer-Verlag, New York, 1979.

7. D. Quillen, On the associated graded ring of a group ring, J. Algebra 10 (1968), 411-418.

8. K. W. Roggenkamp and L. Scott, The isomorphism problem and units in group rings of finite order, in Groups-St. Andrews 1981, London Math. Soc. Lecture Notes, vol. 71, 1982, pp. 313-327.

9. F. Rõhl, On induced isomorphisms of group rings, in Groups-Korea 1983, Lecture Notes in Math., vol. 1098, Springer-Verlag, New York, 1984, pp. 136-141. 
10. R. Sandling, Group rings of circle and unit groups, Math. Z. 140 (1974), 195-202.

11. _ The isomorphism problem for group rings: a survey, in Orders and Their Applications, Lecture Notes in Math., vol. 1142, Springer-Verlag, New York, 1985, pp. 256-288.

12. $\ldots$, The modular group algebra of a central-elementary-by-abelian p-group, Arch. Math. 52 (1989), 22-27.

13. H. N. Ward, Some results on the group algebra of a group over a prime field, Seminar on Finite Groups and Related Topics at Harvard University, 1960-61.

Department of Mathematics, University of Alabama, Tuscaloosa, Alabama 35487 